\title{
The rise of diabetes prevalence in the Arab region
}

\author{
Abdesslam Boutayeb $^{1^{*}}$, Mohamed E. N. Lamlili ${ }^{1}$, Wiam Boutayeb ${ }^{1}$, Abdellatif Maamri ${ }^{2}$, \\ Abderrahim Ziyyat ${ }^{3}$, Noureddine Ramdani ${ }^{3}$ \\ ${ }^{1}$ Laboratory of Stochastic and Determinstic Modelling \& Research Unit Associated to CNRST, Faculty of Sciences, University \\ Mohamed Ier, Oujda, Morocco. \\ ${ }^{2}$ Health Career Training Institute \& Faculty of Sciences, Oujda, Morocco \\ ${ }^{3}$ Department of Biology, Faculty of Science, University Mohamed Ier, Oujda, Morocco \\ Email: *x.boutayeb@menara.ma, Mohamed.lamlili@gmail.com, wiam.boutayeb@gmail.com, abdellatifm@hotmail.com, \\ ziyyat@yahoo.fr, ramdani.noureddine@gmail.com
}

Received 17 March 2012; revised 21 April 2012; accepted 26 April 2012

\begin{abstract}
Introduction: Arab populations have many similarities and dissimilarities. They share culture, language and religion but they are also subject to economic, political and social differences. The purpose of this study is to understand the causes of the rising trend of diabetes prevalence in order to suggest efficient actions susceptible to reduce the burden of diabetes in the Arab world. Method: We use principal component analysis to illustrate similarities and differences between Arab countries according to four variables: 1) the prevalence of diabetes, 2) impaired glucose tolerance (IGT), 3) diabetes related deaths and 4) diabetes related expenditure per person. A linear regression is also used to study the correlation between human development index and diabetes prevalence. Results: Arab countries are mainly classified into three groups according to the diabetes comparative prevalence (high, medium and low) but other differences are seen in terms of diabetes-related mortality and diabetes related expenditure per person. We also investigate the correlation between the human development index (HDI) and diabetes comparative prevalence $(\mathrm{R}=$ 0.81). Conclusion: The alarming rising trend of diabetes prevalence in the Arab region constitutes a real challenge for heath decision makers. In order to alleviate the burden of diabetes, preventive strategies are needed, based essentially on sensitization for a more healthy diet with regular exercise but health authorities are also asked to provide populations with heathcare and early diagnosis to avoid the high burden caused by complications of diabetes.
\end{abstract}

Keywords: Arab Region; Diabetes; Prevalence; Transition; Human Development; Regression; Principal Component Analysis

${ }^{*}$ Corresponding author.

\section{INTRODUCTION}

By the end of 2011, the Arab population was about 340 million representing nearly $5 \%$ of the world population and living in the 22 countries and territories constituting the League of Arab States (Syria being suspended since November 2011). Living in a vast region stretching from North Africa to West Asia. Arab populations have many similarities and dissimilarities. They share culture, language and religion but they are also subject to economic, political and social differences [1,2].

During the last decade, the United Nations Development Program (UNDP) devoted a series of reports to human development in the Arab world. The first Arab Human Development Report (AHDR 2002) diagnosed three cardinal deficits impeding human development in Arab countries (knowledge acquisition, freedom and good governance, and woman's empowerment) [3]. Accordingly, the second report (AHDR 2003) was devoted to the knowledge acquisition deficit whereas the third report (AHDR 2004) dealt with the deficit in freedom and good governance $[4,5]$. The question of woman's empowerment was examined by the fourth report (AHDR 2005) [6]. Finally, a fifth report on "Challenges to Human Security in the Arab Countries" was released in 2009 (AHDR 2009), arguing that the trend in the region has been to focus more on the security of the state than on the security of the people [7]. These reports stimulated an interesting and controversial debate among Arab intellectuals [1,8-12]. In 2009, another series of papers were devoted to the Arab region under the title "health in a troubled region" [2,13-16]. However, very little or no attention was given by the authors to the burden of diabetes and non communicable diseases.

In December 2010 started the "the Arab spring". The first flash was given in Tunisia, followed by a wave of uprisings through the whole Arab region. All the Arab rulers were abruptly shaken by demonstrations and pro- 
tests, generally peaceful. The Arab awakening began by relieving the Tunisian president from his duties then followed the Egyptian, Libyan and Yemeni presidents. In other countries rulers avoided destitution by quickly taking alleviating decisions: in Morocco a constitutional reform was undertaken followed by anticipated elections, in Saudi Arabia women were granted the right to vote (although it will not be effective until 2015), in Algeria, Jordan, Kuwait and Oman protesters imposed increases in the minimum wage, job opportunities and freedom.

In December 2011, another alarm awakened Arab governments when International Diabetes Federation announced the last diabetes estimates at the fifth conference held in Dubai. Six of the top 10 countries with the highest prevalence of diabetes (in adults aged 20 to 79 years) are in the Middle East: Kuwait (21.1\%), Lebanon (20.2\%), Qatar (20.2\%), Saudi Arabia (20.0), Bahrain (19.9\%) and UAE (19.2\%) [17].

The main purpose of this study is to understand the causes of the rising trend of diabetes prevalence in order to suggest efficient actions susceptible to reduce the burden of diabetes in the Arab world. A comparison is made between different Arab countries according to four variables: prevalence of diabetes, impaired glucose tolerance (IGT), diabetes related deaths and diabetes related expenditure per person.

\section{METHOD}

\subsection{Data Sources}

We used the recent data on diabetes as published by the International Diabetes Federation (IDF) in 2011 [17]. However, the figures released by IDF are based on estimates published by different authors and organisms related to studies carried out in different countries and regions. In the absence of reliable national data in many countries, IDF used extrapolations either from neighboring countries or from local to national level [18]. Consequently, IDF data were compared with, end eventually completed by the most recent data released by Arab governments and/or published by researchers in reviewed journals for the whole region [19-21] and separately for different Arab countries (Algeria [22], Egypt [23], Jordan [24], Morocco [25-27], Saudi Arabia [2830], Sudan [31], UAE [32]).

\subsection{Variables}

For Principal Component Analysis (PCA), the constraints on availability of data for the whole Arab region lead as to concentrate on four health variables, namely: 1) diabetes comparative prevalence according to WHO standards, as published by IDF, 2) IGT prevalence, 3) diabetes related deaths, and 4) mean diabetes-related expenditure per person with diabetes, expressed in USD.

For the linear regression, we used Human Development Index (DHI) as an explicative variable and diabetes comparative prevalence as an explained variable. The HDI values used were those published by the United Nations Development Program (UNDP) for the year 2011 [33].

\section{RESULTS AND DISCUSSION}

\subsection{Diabetes: A Worldwide Silent Epidemic}

Diabetes is a non communicable chronic disease in which the body is unable to produce insulin or cannot properly use the insulin it produces. Consequently, the system is no longer self-regulated and the level of sugar in the blood goes beyond the limits (threshold). Repeated and prolonged episodes of hyperglycemia can damage blood vessels and nerves, leading to the well known devastating diabetes complications (retinopathy, neuropathy, nephropathy, amputation and cardiovascular disease).

According to International Diabetes Federation, diabetes is classified into three main types: 1 ) Type 1 diabetes (formerly known as insulin dependent diabetes or juvenile diabetes) which affects young people and necessitates treatment with insulin and diet; 2) Type 2 diabetes (previously known as non insulin dependent diabetes or adult diabetes) which affects generally people after 40 age years; 3) Gestational diabetes mellitus (GDM) which develops during pregnancy and affects $2 \%$ to $4 \%$ of all pregnancies. It is worth noting the high proportions of people who have developed diabetes but remain undiagnosed and those with blood glucose higher than normal, but not high enough to be diagnosed as type 2 diabetes (pre-diabetes stage) also said to have impaired glucose tolerance (IGT) or impaired fasting glucose (IFG). IFG being defined as high blood glucose after a fast (glycaemia between 1.1 and $1.26 \mathrm{~g} / \mathrm{l}$ ), whereas IGT is defined as high blood glucose levels after eating. People in the prediabetes stage have a higher risk of developing type 2 diabetes, especially in presence of obesity and some inability of the body to use the insulin it produces [17].

During the last decades, the world has seen a dramatic increase in the prevalence of diabetes. This chronic disease affects not only health of people living with it, but also imposes significant direct and indirect costs on them, on their families and relatives, and on the whole society. The growing burden of diabetes is challenging health decision makers in developed and developing countries alike but its impact is more devastating in low income countries. The recent figures released by the International Diabetes Federation (IDF) are alarming. Worldwide, it is estimated that 366 million people have diabetes and half of them are not aware that they have the dis- 
ease (undiagnosed). If the current trend is maintained and no urgent action is taken, the number of people living with diabetes is expected to reach 552 million by 2030 . Although the number of people with diabetes is increaseing in every country, about $80 \%$ are in low- and middle-income countries $[17,18]$.

\subsection{Prevalence of Diabetes in the Arab Region}

According to the last figures released by the International Diabetes Federation (IDF), a rising trend of incidence and prevalence is seen in every country around the world. However, the Arab region appears to have a higher prevalence of diabetes than the global average. The Middle East and North Africa region has the highest comparative prevalence (11\%). Six of the top 10 countries with the highest prevalence of diabetes (in adults aged 20 to 79 years) are in the Arab region: Kuwait (21.1\%), Lebanon (20.2\%), Qatar (20.2\%), Saudi Arabia (20.0), Bahrain (19.9\%) and UAE (19.2\%). For the 20 Arab countries for which data is available, nearly 20.5 million people are living with diabetes and another 13.7 million are in the pre-diabetes stage, having impaired Glucose tolerance (IGT). While in developed countries most people with diabetes are above the age of retirement, in the Arab region nearly three quarters (73.4\%) of diabetics are under 60 years of age and in the peak of their productive years, which makes the burden in terms of disability due to diabetes heavier [17].

\subsection{The Burden of Diabetes in Arab Countries}

In the Arab region, the number of deaths attributed to diabetes is about 170,000 adult people, representing more than $10 \%$ of all deaths in the region. However, beyond mortality, temporary and permanent disabilities are often caused by complications of diabetes such as blindness, amputations, kidney failure and cardio vascular diseases (CVDs). Consequently, diabetes causes an important economic burden due to the cost of treatment and the loss of productivity. According to IDF estimates, the Arab region was expected to spend USD 8.7 billion as expenditure for diabetes in 2011. However, the total healthcare expenditures due to diabetes in the region account for less than $2 \%$ of the total global figure whereas the number of diabetics in the region (20.5 million) represents $5.6 \%$ of the world total number of diabetes (366 million). According to a recent study carried out on the direct medical cost of diabetes and its complications in the Eastern District of Abu Dhabi emirate (Al-Ain region), the annual direct treatment costs of a diabetic patient without complications (US\$ 1605) was 3.2 times higher than the per capita expenditure for health care in the UAE (US\$ 497). The cost increased notably with the presence of micro-vascular complications (2.2 times) and macro-vascular complications (6.4 times). In patients with both micro- and macro-vascular complications, the treatment cost was 9.4 times higher [33].

\subsection{Similarities and Differences between Arab Countries}

Principal Components Analysis (PCA) is used to summarise the information and get an easy comparison between Arab countries after projection of variables and countries on the first plan given by factors F1 and F2. The corresponding eigenvalues give the percentages of information explained by these factors respectively. In our case, the first and second axes explain $62 \%$ and $27 \%$ respectively. Consequently, we have a good representation on the first plan $(\mathrm{F} 1 \times \mathrm{F} 2)$ which summarises $90 \%$ of information. Additional information may be obtained by the third and fourth axis, but the interpretation is then conditional to what was given by the precedent factors.

We get an interesting sketch by projecting the four variables and the 20 countries on the first plane $(\mathrm{F} 1 \times$ F2).

Figure 1 shows that the first axis (F1) is mainly determined by the variables prevalence, cost of diabetes and IGT which are correlated between each other, while the second axis (F2) is mainly determined by the variable diabetes death.

As indicated in Figure 2, the projection of countries on the first plan provides a first classification into three groups according to the level of diabetes comparative prevalence. A first group (G1) with seven countries (Bahrain (19.88), Egypt (16.94), Kuwait (21.10), Lebanon (20.17), Qatar (20.17), Saudi Arabia (20.02) and UAE (19.22)), characterised by high prevalence of diabetes. A second group of seven countries with medium prevalence (Iraq (9.33), Jordan (12.37), Oman (10.75), Sudan (8.72), Syria (10.15), Tunisia (9.68) and Yemen (9.87)). And finally a third group of six countries with relatively low prevalence (Algeria (7.04), Comoros (8.57), Djibouti (6.4), Mauritania (4.36), Morocco (6.96) and Somalia (4.26)).

In the first group, a second separation is provided by the second axis which clearly opposes countries with low rates of diabetes related deaths (Bahrain(5.5), Kuwait (4.3), Qatar (3.0) and UAE (2.3)) to countries with high rates of diabetes related deaths (Egypt (13.5), Lebanon (16.2) and Saudi Arabia (9)).

At a lesser extent, similar opposition between countries in the second or in the third group can be seen according to the second axis. For instance, Oman and Sudan have nearly the same combined prevalence of diabetes and IGT but the cost of diabetes per person is five times higher in Oman while the rate of diabetes related death is three times higher in Sudan. Similarly, Maurita- 


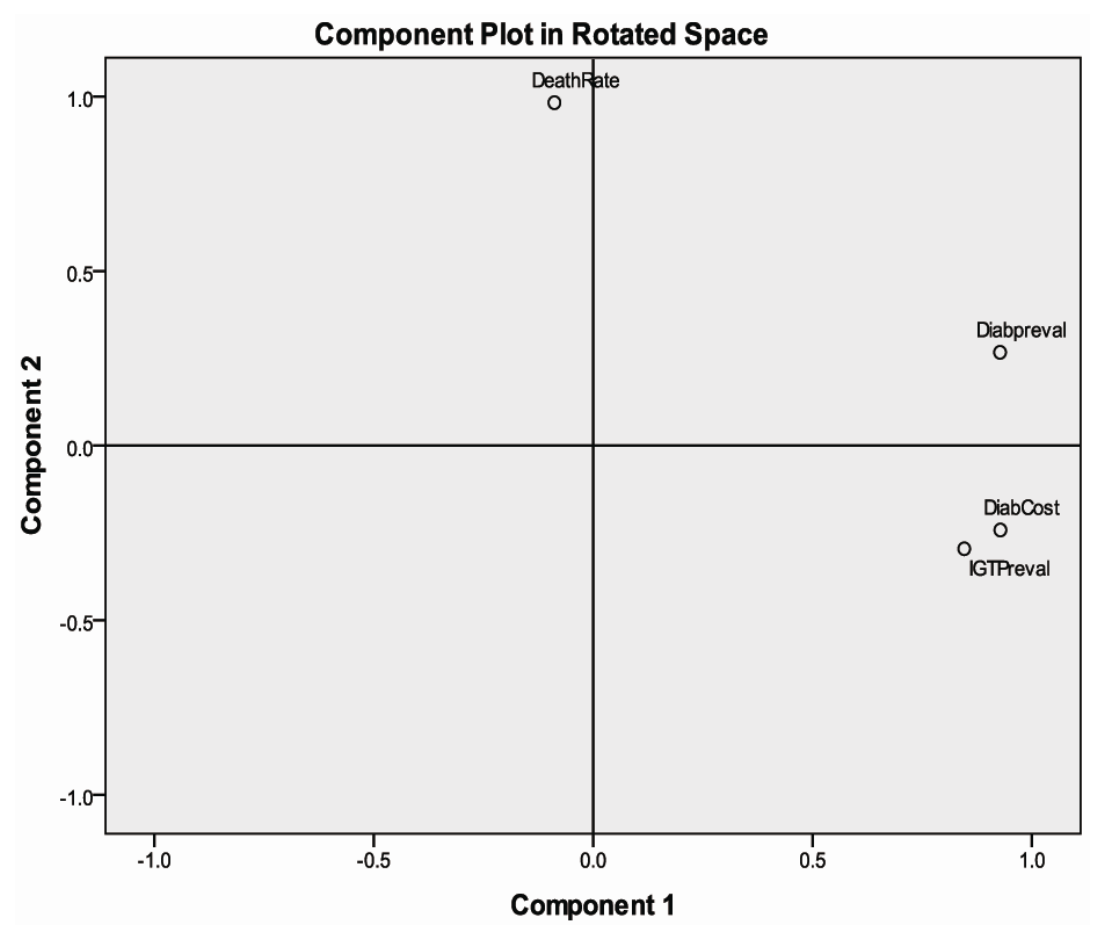

Component1: first axis given by PCA; Component2: second axis given by PCA.

Figure 1. Projection of variables on the first plan given by Principal Component Analysis (PCA).

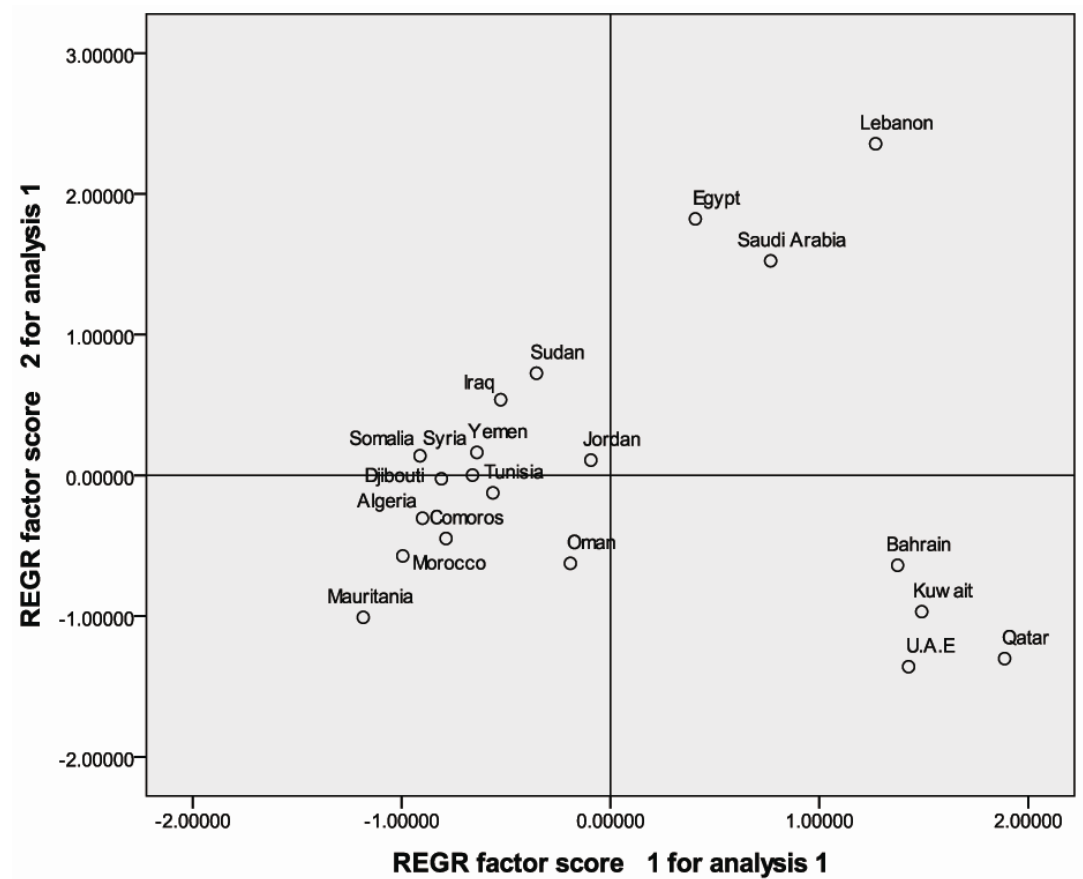

REGR factor score1 for analysis1: first axis given by PCA; REGR factor score2 for analysis1: second axis given by PCA.

Figure 2. Projection of countries on the first plan given by Principal Component Analysis (PCA).

nia and Somalia have nearly the same combined prevalence of diabetes and IGT but the cost of diabetes per person is double in Mauritania while the rate of diabetes related death is 2.5 times higher in Somalia. 


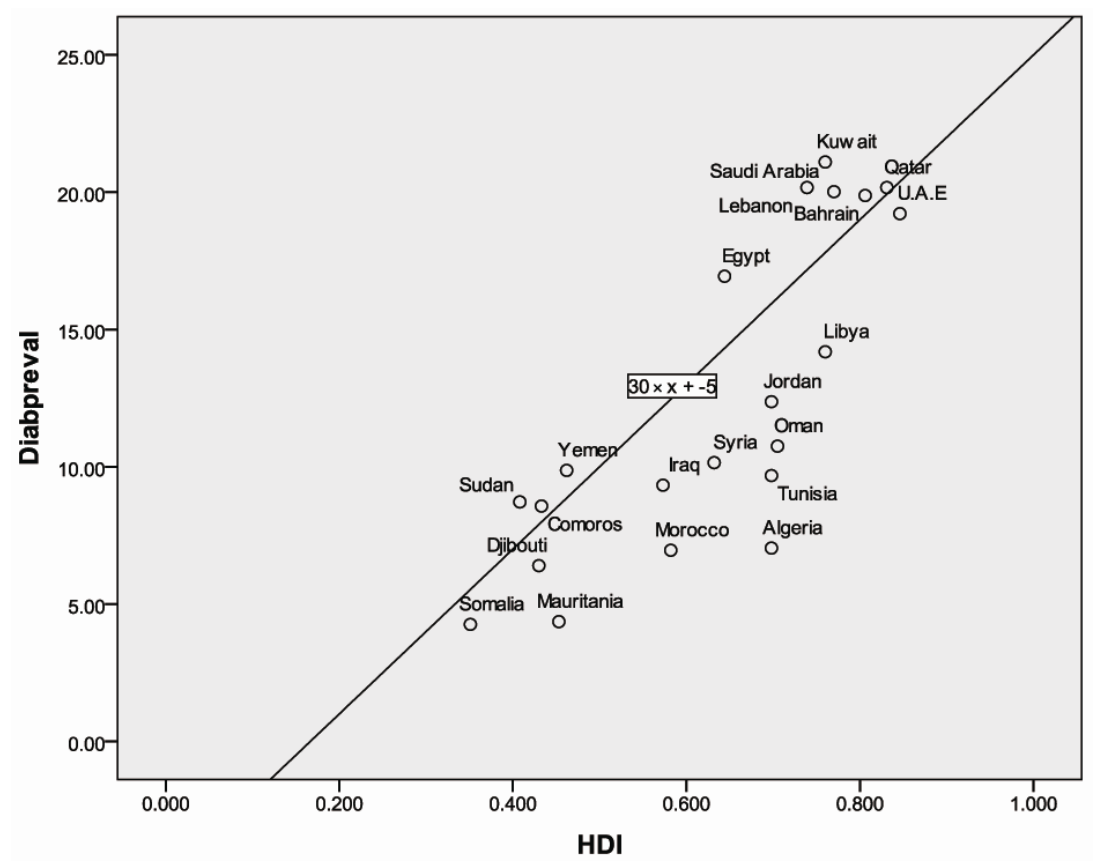

Diabpreval: diabetes comparative prevalence; HDI: human development index.

Figure 3. Linear regression showing correlation between diabetes prevalence and HDI.

\subsection{Correlation between Human Development Index and Prevalence of Diabetes}

In the Arab region, diabetes prevalence seems to increase with human development. To check this hypothesis, we carried out a linear regression with diabetes prevalence as explained variable (Diabpreval) and human development index (HDI) as explicative variable. As indicated by Figure 3, a relatively high correlation $(\mathrm{R}=0.81)$ is seen between the two variables. The figure also indicates clearly the extreme opposition between the group of six countries with the highest HDI and highest prevalence of diabetes (Kuwait (0.76, 21.1\%), Lebanon (0.739, 20.2\%), Qatar (0.831, 20.2\%), Saudi Arabia (0.77, 20.0), Bahrain (0.806, 19.9\%) and UAE (0.846, 19.2\%) ) and another group of six countries with low HDI and low diabetes prevalence (Somalia $(0.351,4.26)$, Mauritania (0.453, 4.36), Djibouti $(0.43,6.4)$, Comoros $(0.433,8.57)$, Sudan $(0.408,8.72)$, Yemen $(0.462,9.87))$. The remaining nine countries have a combined HDI and diabetes prevalence between those of the lowest and highest groups. (Libya $(0.76,14.19)$, Jordan $(0.698,12.37)$, Oman $(0.705,10.75)$, Tunisia (0.698, 9.68), Algeria (0.698, 7.04), Syria (0.632, 10.15), Egypt (0.644, 16.94), Morocco (0.582, 6.96), Iraq $(0.573,9.33))$.

\section{CONCLUSIONS}

The majority of Arab countries are engaged in a multidimensional transition (demographic, economic, epidemiological and geographic). This 4-dimensional transi- tion engendered many direct and indirect factors explaining the high prevalence of diabetes. The shift from rural to urban dominance lead to sedentary life and less physical exercise. The socioeconomic development allowed for higher income and more consumption especially of fast-food and western diet. Socio-cultural habits and Arab generosity (Arab people enjoy inviting guests to their homes for meals) encouraged gathering and high calorie intake. Finally, with epidemiological and demographic transitions, ageing with a different history of early childhood conditions fostered the development of obesity and impaired glucose tolerance.

The rising rates of obesity, IGT and diabetes constitute a real challenge in the Arab region.

In order to alleviate the burden of diabetes, preventive strategies are needed, based essentially on sensitization for a more healthy diet with regular exercise but health authorities are also asked to provide populations with heath-care and early diagnosis to avoid the high burden caused by complications of diabetes in terms of mortality and morbidity causing loss of economic productivity.

\section{REFERENCES}

[1] Boutayeb, A. and Serghini, M. (2006) Health indicators and human development in the Arab region. International Journal of Health Geographics, 5, 61. doi:10.1186/1476-072X-5-61

[2] Mandil, A. (2009) Mosaic Arab world, health and development. International Journal of Public Health, 54, 361- 


\section{2. doi:10.1007/s00038-009-0065-3}

[3] Arab Human Development Report (2002) Creating opportunities for future generations.

http://www.arab-hdr.org/publications/other/ahdr/ahdr200 2e.pdf

[4] Arab Human Development Report (2003) Building a knowledge society.

http://www.arab-hdr.org/publications/other/ahdr/ahdr200 3e.pdf

[5] Arab Human Development Report (2004) Towards freedom in the Arab world.

http://www.arab-hdr.org/publications/other/ahdr/ahdr200 $\underline{\text { 4e.pdf }}$

[6] Arab Human Development Report (2005) Towards the rise of women in the Arab world. http://www.arab-hdr.org/publications/other/ahdr/ahdr200 $\underline{\text { 5e.pdf }}$

[7] Arab Human Development Report (2009) Challenges to human security in the Arab countries.

http://www.arab-hdr.org/publications/other/ahdr/ahdr200 9e.pdf

[8] Baroudi, S.E. (2002) The 2002 Arab human development report: Implications for democracy. Middle East Policy, 11, 132-14. doi:10.1111/j.1061-1924.2004.00146.x

[9] Jabbour, S. (2002) Critical reflections on health and development in the Arab world. Newsletter of the Economic Research Forum for the Arab Counties, Iran \& Turkey, 9, 24-27.

[10] Jabbour, S. (2003) Health and development in the Arab world: Which way forward? British Medical Journal, 326, 1141-1143. doi:10.1136/bmj.326.7399.1141

[11] Fergani, N. (2003) Second Arab human development report: The need for a knowledge society. Newsletter of the Economic Research Forum for the Arab Counties, Iran and Turkey, 10, 10-11.

[12] Boutayeb, A. (2006a) Social inequalities and health equity in Morocco. International Journal for Equity in Health, 5, 1. doi:10.1186/1475-9276-5-1

[13] Maziak, W. (2009a) The crisis of health in a crisis ridden region. International Journal of Public Health, 54, 349355. doi:10.1007/s00038-009-0061-7

[14] Maziak, W. (2009b) Commentary: Is it light at the end of the tunnel, or another train coming? Concluding comments. International Journal of Public Health, 54, 363364. doi:10.1007/s00038-009-0067-1

[15] Nuwayhid, I. (2009) How to break the cycle of hopelessness? International Journal of Public Health, 54, 357358. doi:10.1007/s00038-009-0062-6

[16] El-Zein, A. (2009) On approaching health in the Arab world. International Journal of Public Health, 54, 359360. doi:10.1007/s00038-009-0063-5

[17] International Diabetes Federation (2011) IDF Diabetes Atlas. 15th Edition, International Diabetes Federation, Brussels.

[18] Guariguata, L., Whiting, D., Weil, C. and Unwin, N. (2011) The international diabetes federation diabetes atlas methodology for estimating global and national preva- lence of diabetes in adults. Diabetes Research and Clinical Practice, 94, 322-332.

doi:10.1016/j.diabres.2011.10.040

[19] Alhyas, L., McKay, A. and Balasanthiran, A. (2011) Prevalence of overweight, obesity, hyperglycaemia, hypertension and dislipdaemia in the gulf: Systematic review. Journal of the Royal Society Medicine, 2, 55. doi:10.1258/shorts.2011.011019

[20] Badran, M. and Laher, I. (2011) Obesity in ArabicSpeaking countries. Journal of Obesity, 2011, Article ID: 686430.

[21] Ramahi, T.M. (2010) CVD in the Asia MER: Global trends and local implications. Asia-Pacific Journal of Public Health, 22, 83. doi:10.1177/1010539510373034

[22] Zaoui, S. and Biémont, C. (2007) Approche épidémiologique du diabète en milieu rural dans la région de Tlemcen (ouest algérien). Cahiers santé, 17, 15-21.

[23] Egypt Ministry of Health (2006) Community based study on non communicable diseases and their risk factors in Egypt. Population and World Health Organization.

[24] Jordan Ministry of Health (2004) Jordan STEAPS survey report, technical report. World Health Organization.

[25] Ramdani, N., Vanderpas, J., Boutayeb, A. et al. (2011) Diabetes and obesity in the eastern Morocco.

http://www.springerlink.com/content/g21m98683313mr6 $\underline{0 /}$

[26] Tazi, M.A., Abir-Khalil, S., Chaouki, N., Cherqaoui, S., Lahmouz, F., Srairi, J.E., et al. (2003). Prevalence of the main cardiovascular risk factors in Morocco: Results of a national survey, 2000. Journal of Hypertension, 21, 897903. doi:10.1097/00004872-200305000-00013

[27] Rguibi, M. and Belahsen, R. (2004) Metabolic syndrome among Moroccan Sahraoui adult women. American Journal of Human Biology, 16, 598-601. doi:10.1002/ajhb.20065

[28] Al-Nuaim, A.R. (1997) Prevalence of glucose intolerance in urban and rural communities in Saudi Arabia. Diabetic Medicine, 14, 595-602.

doi:10.1002/(SICI)1096-9136(199707)14:7<595::AID-DI A377>3.0.CO;2-C

[29] El Hazmi, M.A., Warsy, A., Al-Swailem, A., Al-Swailem, A. and Sulaimani, R. (1998) Diabetes mellitus as a health problem in Saudi Arabia. Eastern Mediterranean Health Journal, 4, 58-67

[30] Al-Nozha, M.M., Al-Maatouq, M.A., Al-Mazrou, Y.Y., et al. (2004) Diabetes mellitus in Saudi Arabia. Saudi Medical Journal, 25, 1603-1610.

[31] ElRayah-Eliadarous, H. (2007) Economic burden of diabetes on patients and their families in Sudan. Karolinska Institutet, Stockholm.

[32] Al-Maskari, F., El-Sadig, M. and Nagelkerke, N. (2010) Assessment of the direct medical costs of diabetes mellitus and its complications in the United Arab Emirates. BMC Public Health, 10, 679. doi:10.1186/1471-2458-10-679

[33] United Nations Development Programme (2011) Human development report. http://hdr.undp.org/en/statistics/ 